\title{
Increased serotonin transporter immunoreactivity intensity in the ileum of patients with irritable bowel disease
}

\author{
INGVILD WENDELBO $^{1,2}$, TAREK MAZZAWI ${ }^{1,2}$ and MAGDY EL-SALHY ${ }^{1,2}$ \\ ${ }^{1}$ Section for Gastroenterology, Department of Medicine, Stord Helse-Fonna Hospital, Stord 5416; \\ ${ }^{2}$ Section for Gastroenterology, Department of Clinical Medicine, University of Bergen, Bergen 5020, Norway
}

Received April 24, 2013; Accepted October 22, 2013

DOI: $10.3892 / \mathrm{mmr} .2013 .1784$

\begin{abstract}
Irritable bowel syndrome (IBS) is a common chronic gastrointestinal disorder, which represents an economic burden to society and considerably reduces the quality of life of patients. In a previous study, the density of serotonin cells in the ileum of IBS patients was lower compared with control subjects. The present study aimed to further investigate the immunoreactivity intensity of serotonin and serotonin-selective reuptake transporter (SERT) in the ileum of IBS patients. A total of 98 patients (77 females and 21 males; mean age, 35 years; range, 18-66 years), which fulfilled Rome III Criteria for IBS, were included in the study. This cohort included 35 patients with diarrhoea-predominant (IBS-D), 31 patients with mixed diarrhoea and constipation (M-IBS) and 32 patients with constipation-predominant (IBS-C) symptoms. A total of 27 subjects were included as controls (16 females and 11 males; mean age, 52 years; range, 20-69 years). Ileal biopsy specimens were immunostained using the avidin-biotin (ABC) complex method for serotonin and SERT. The immunoreactivity intensity was quantified by computerised image analysis using Olympus cellSens imaging software. No statistical difference of serotonin immunoreactivity intensity was identified in multiple comparisons between controls, IBS-total, IBS-D, IBS-M and IBS-C. The SERT immunoreactivity intensity was significantly increased in IBS patients as compared with controls, regardless of the subtype. It was concluded that the increase in ileal epithelial content of SERT increases the intracellular uptake of serotonin and its degradation in the gut epithelial cells and consequently decreases the availability of serotonin
\end{abstract}

Correspondence to: Professor Magdy El-Salhy, Section for Gastroenterology, Department of Medicine, Stord Helse-Fonna Hospital, Tysevegen 64, Stord 5416, Norway

E-mail: magdy.el-salhy@helse-fonna.no

Key words: diarrhoea, constipation, irritable bowel syndrome, immunohistochemistry, serotonin, serotonin-selective reuptake transporter within the gut mucosa. The low availability of serotonin at its receptors occurred in all IBS subtypes. This may indicate that this abnormality is associated with a common symptom in all IBS subtypes, namely abdominal pain/discomfort. Serotonin acts upon sensory neurons in the submucosal and myenteric ganglia, as well as in the spinal cord, which is in agreement with this hypothesis.

\section{Introduction}

Irritable bowel syndrome (IBS) is a common chronic gastrointestinal disorder (1-9). It occurs primarily at a young age, with a female predominance and causes lifelong illness (2,10-21). IBS considerably reduces the quality of life of the patients due to the uncomfortable symptoms associated with the syndrome, which are often found embarrassing and humiliating and thus interfere with education, working ability and social interactions. Furthermore, IBS represents an economic burden to society caused by overconsumption of healthcare resources and lower work productivity of IBS patients $(9,13,20,22-24)$.

The diagnosis of IBS is based primarily on symptom assessment, including the Rome III criteria $(25,26)$. Patients with IBS suffer from abdominal discomfort or pain, altered bowel habits and bloating/abdominal distension $(1,2)$. Based on the stool pattern, patients with IBS are subdivided, according to Rome III criteria, into four subtypes, namely diarrhoea-predominant (IBS-D), constipation-predominant (IBS-C), mixed diarrhoea and constipation (M-IBS) and unclassified IBS (U-IBS) $(25,26)$.

Serotonin producing cells occur in large numbers in the mucosa of the majority of segments of the gastrointestinal tract and represent the main source of serotonin in the human (27-29). Serotonin acts upon sensory and motor neurons in the submucosal and myenteric ganglia as well as in the spinal cord (27). This, in turn, stimulates motor, secretory and vasodilatory reflexes as well as afferent signals to the central nervous system (27). Serotonin, following acting on its receptors, is transported by serotonin-selective reuptake transporter (SERT) into gut epithelial cells, where it is degraded $(27,30)$. The gut mucosa has a high capacity for the production of SERT as all the epithelial cells lining the luminal surface of the gut express SERT $(29,31)$. Serotonin cell densities in the ileum and colon were reduced in IBS 
patients compared with those in the duodenum and rectum which were unchanged (32-36). However, SERT immunoreactivity intensity has been observed to be high in the rectum $(36,37)$.

In the present study the immunoreactivity intensity of serotonin and SERT was investigated in the ileum of the same cohort of IBS patients where low density of ileal serotonin cells were found (32).

\section{Materials and methods}

Patients and controls. Ninety-eight patients (77 females and 21 males; mean age, 35 years; range, 18-66 years), which fulfilled Rome III Criteria for IBS were included in the study $(25,38)$. The IBS subtypes were distributed as follows: 35 patients with IBS-D, 31 patients with IBS-M and 32 patients with IBS-C. Symptoms had been present in all patients for a number of years and the onset of IBS symptoms were not associated with any events, in particular gastrointestinal infections. All patients underwent a complete physical examination and were investigated using the following blood tests: Full blood count, electrolytes, inflammatory markers, liver tests and thyroid function tests. Patients also underwent further gastroscopy with duodenal biopsies, which were used to exclude celiac disease.

A total of 27 subjects who underwent colonoscopy (16 females and 11 males; mean age, 52 years; range, 20-69 years) were used as controls. These subjects underwent a colonoscopy for the following reasons: Gastrointestinal bleeding, where the source of bleeding was identified as haemorrhoids $(n=18)$ or angiodysplasia $(n=2)$ and health worries resulting from a relative being diagnosed with colon carcinoma $(n=7)$.

The study was performed in accordance with the Declaration of Helsinki and was approved by the Regional Committee for Medical and Health Research Ethics, Health West, University of Bergen (Bergen, Norway). All subjects provided oral and written consent.

Colonoscopy, histopathology and immunohistochemistry. Colonoscopy was performed on the patients and controls, segmental biopsy specimens were taken from the colon and rectum and four biopsy samples were extracted from the ileum of each subject. These biopsy samples were fixed overnight in $4 \%$ buffered paraformaldehyde, embedded in paraffin and sliced into $5-\mu \mathrm{m}$ sections. The sections were stained with hematoxylin and eosin (H\&E) and immunostained by the avidin-biotin complex (ABC) method using the Vectastain ABC kit (Vector Laboratories, Burlingame, CA, USA). The sections were hydrated and immersed in phosphate-buffered saline buffer (PBS; pH 7.4) and were incubated with the primary antibodies at room temperature for $2 \mathrm{~h}$. The sections were washed in PBS buffer and incubated with biotinylated swine anti-mouse IgG (Vector Laboratories) diluted 1:100 for $30 \mathrm{~min}$ at room temperature. Following washing the slides in PBS buffer, the sections were incubated for $30 \mathrm{~min}$ with avidin-biotin-peroxidase complex diluted 1:100 and immersed in 3,3'-diaminobenzidine (DAB) peroxidase substrate (Vector Laboratories), followed by counterstaining in hematoxylin. The primary antibodies used were

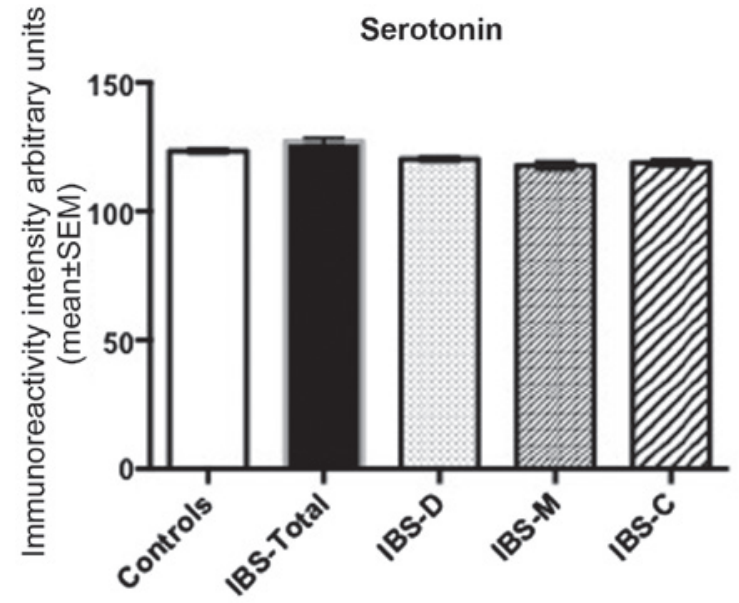

Figure 1. Serotonin immunoreactivity intensity in controls, IBS-total, IBS-D, IBS-M and IBS-C. IBS, irritable bowel syndrome; IBS-D, diarrhoea-predominant; IBS-C, constipation-predominant; M-IBS, mixed diarrhoea and constipation, U-IBS unclassified IBS.

monoclonal mouse anti-serotonin (code no. 5HT-209; Dako, Carpinteria, CA, USA) and mouse anti-human serotonin transporter (code no. GR120164-1; Abcam, Cambridge, MA, USA). The antibodies were used at dilutions of 1:1,500 and 1:500, respectively.

Computerised image analysis. The immunoreactivity intensities of serotonin and SERT were measured using Olympus cellSens imaging software (version 1.7). A x20 magnification was used, for which each frame (field) displayed on the monitor represented an area of $0.14 \mathrm{~mm}^{2}$ of the tissue. In each individual, serotonin and SERT were measured in five randomly selected fields. Immunostained sections from IBS patients and controls were coded and mixed and all measurements were assessed by the same person without knowledge of the identity of the sections.

Statistical analysis. Differences between controls, all IBS patients (IBS-total) and IBS-D, IBS-M and IBS-C patients were analysed by the Kruskal-Wallis nonparametric test with Dunn's test as a post-test. The data are presented as the mean \pm standard error of the mean values and $\mathrm{P}<0.05$ was considered to indicate a statistically significant difference.

\section{Results}

Endoscopy and histopathology. The ileum, colon and rectum of the patients and the control subjects were macroscopically normal. The results of histopathological examinations of the ileum, colon and rectum were normal in the patients and controls.

Immunohistochemistry and computerised image analysis. In the patients and control subjects, serotonin-immunoreactive cells were found primarily in the intestinal crypts. These cells were basket- or flask-shaped. SERT immunoreactivity was observed in the lining epithelium of the ileum in the patients and controls. 


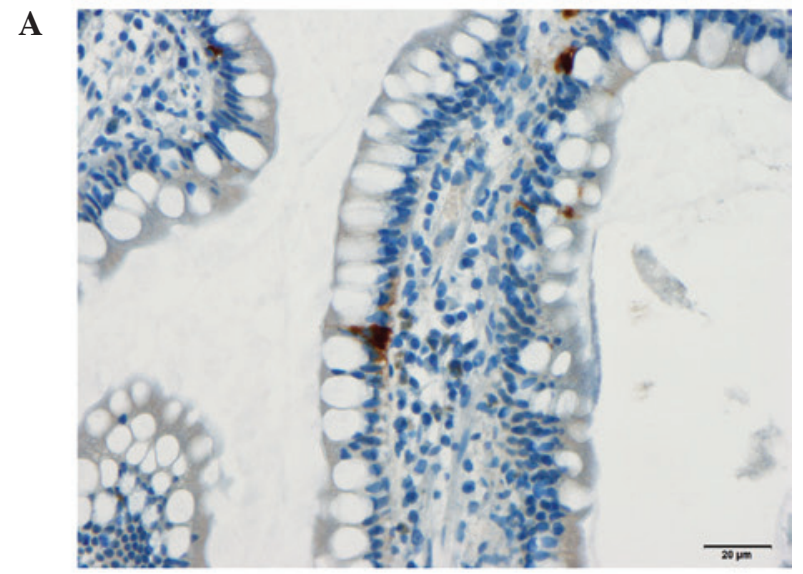

B

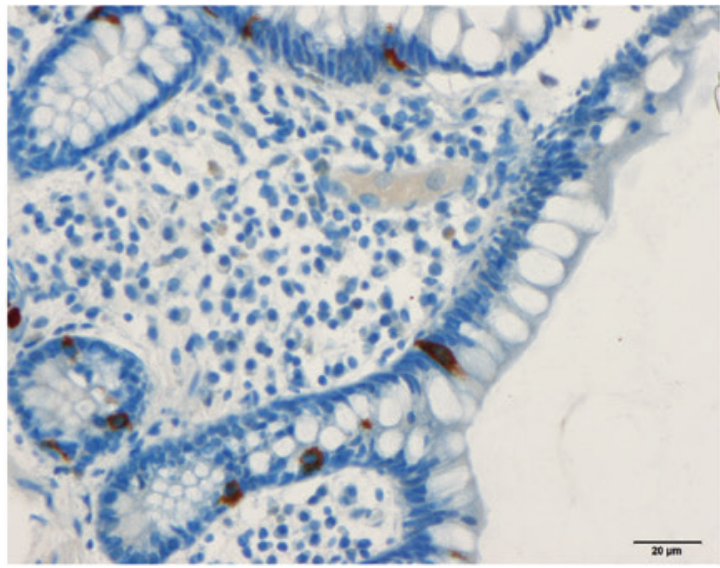

C

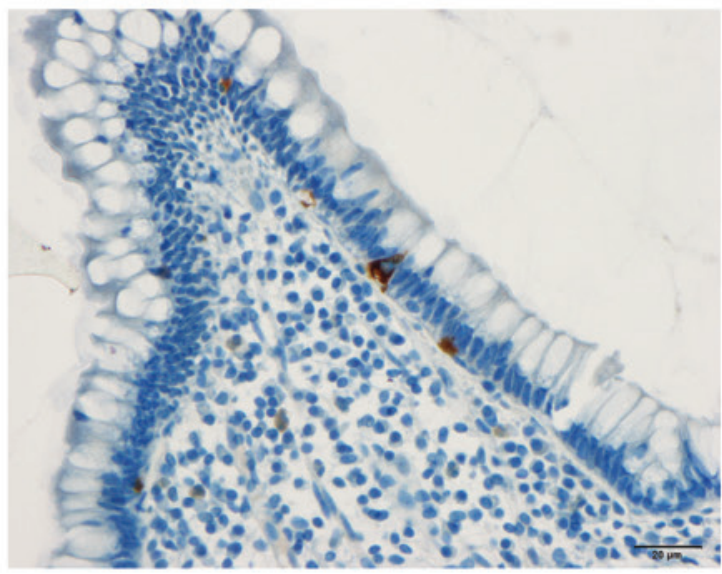

D

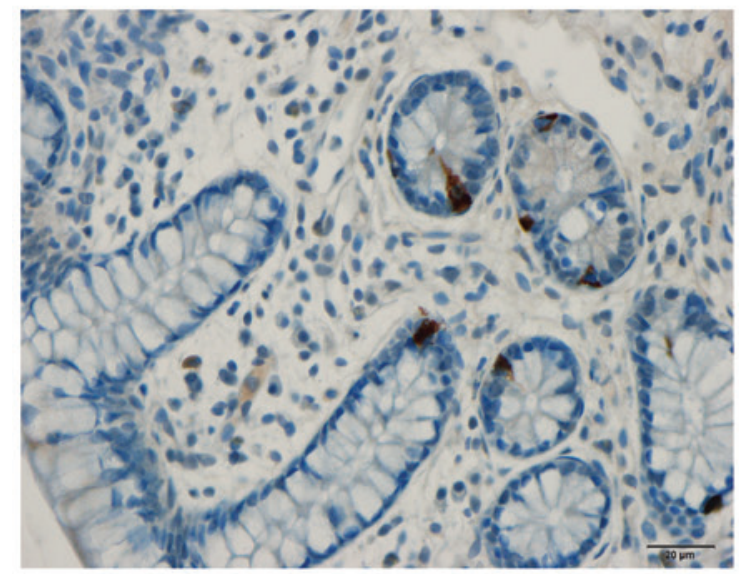

Figure 2. Serotonin immunoreactive cells in the ileum of (A) a control subject and in patients with (B) IBS-D, (C) IBS-M and (D) IBS-C. ABC staining, magnification x40. IBS, irritable bowel syndrome; IBS-D, diarrhoea-predominant; IBS-C, constipation-predominant; M-IBS, mixed diarrhoea and constipation.
$\mathbf{A}$

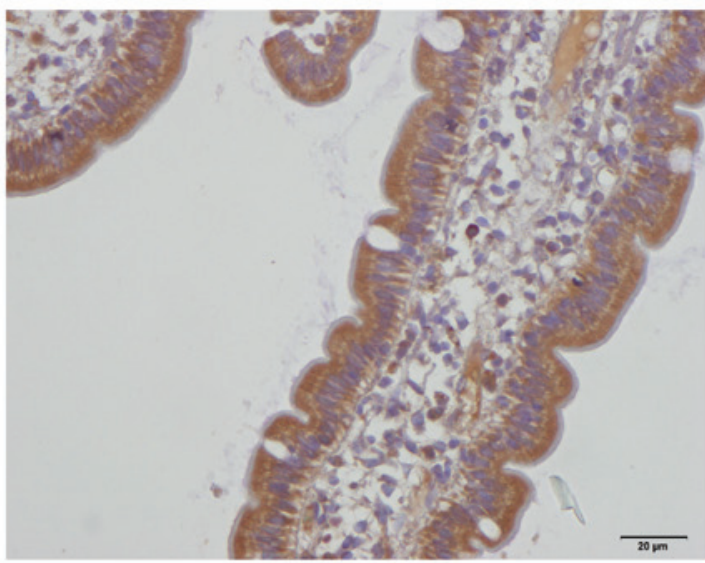

B

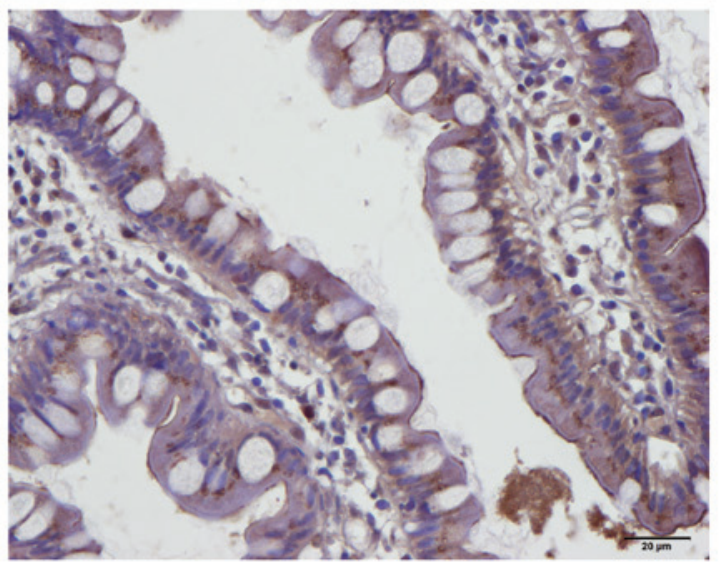

C

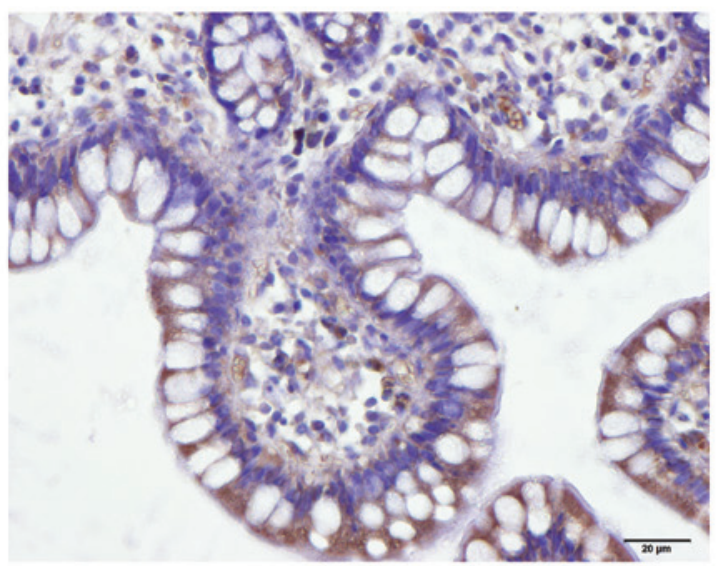

D

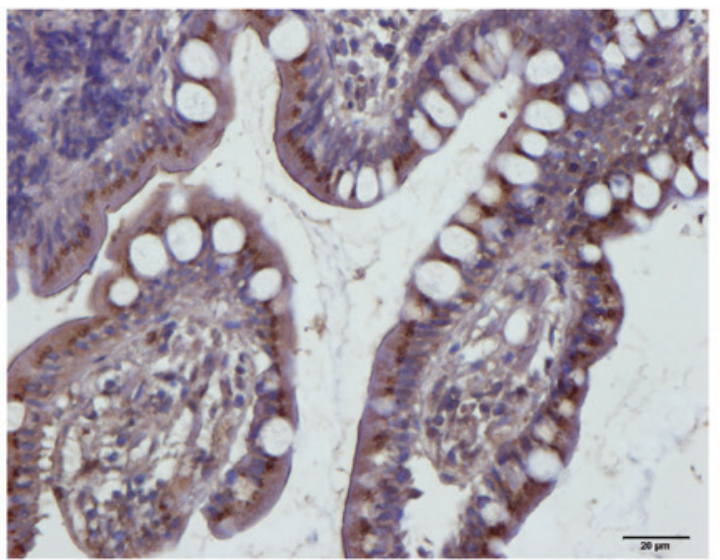

Figure 3. SERT immunoreactivity in the epithelial cells lining the ileum lumen in (A) a control subject and in patients with (B) IBS-D, (C) IBS-M and (D) IBS-C. ABC staining, magnification $\mathrm{x} 40$. SERT, serotonin-selective reuptake transporter; IBS, irritable bowel syndrome; IBS-D, diarrhoea-predominant; IBS-C, constipation-predominant; M-IBS, mixed diarrhoea and constipation. 


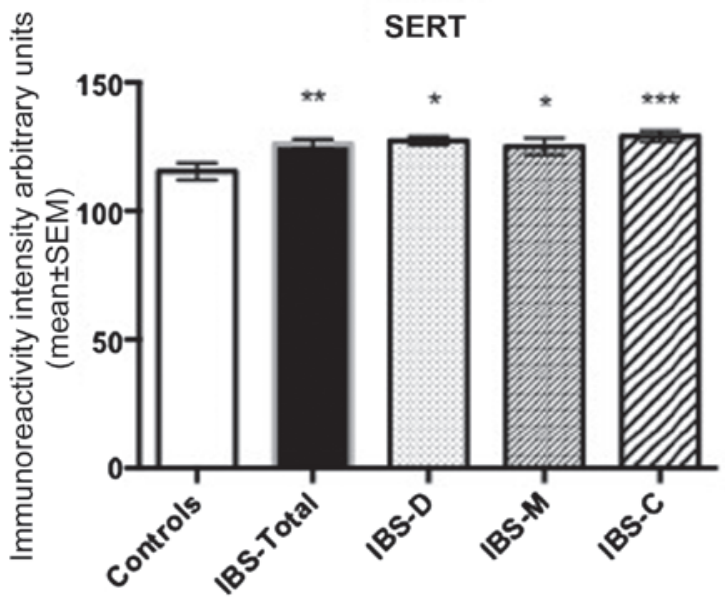

Figure 4. SERT immunoreactivity intensity in ileum epithelial cells of control subjects, IBS-total, IBS-D, IBS-M and IBS-C. ${ }^{*} \mathrm{P}<0,05,{ }^{* *} \mathrm{P}<0.01$ and ${ }^{* * *} \mathrm{P}<0.0001$. IBS, irritable bowel syndrome. SERT, Serotonin-selective reuptake transporter; IBS-D, diarrhoea-predominant; IBS-C, constipation-predominant; M-IBS, mixed diarrhoea and constipation.

Serotonin immunoreactivity intensity. The immunoreactivity intensity of serotonin in the controls was $121.7 \pm 0.8$. The corresponding figures for IBS-total, IBS-D, IBS-M and IBS-C were $122.9 \pm 2.0,120.3 \pm 1.1,117.8 \pm 1.7$ and $118.9 \pm 1.3$, respectively. There was no statistical difference in multiple comparisons between controls, IBS-total, IBS-D and IBS-C $(\mathrm{P}=0.3)$. The post-test did not reveal any statistical differences between the four groups $(\mathrm{P}<0.05$ in all; Figs. 1 and 2$)$.

SERT immunoreactivity intensity. The immunoreactivity intensity of SERT in controls, IBS-total, IBS-D, IBS-M and IBS-C were $115.4 \pm 3.3,126.1 \pm 1.9,127.3 \pm 1.6,125.1 \pm 3.2$ and 129.1 \pm 2.1 , respectively (Figs. 3 and 4). There was a significant statistical difference in multiple comparisons between controls, IBS-total, IBS-D, IBS-M and IBS-C $(\mathrm{P}=0.0005)$. SERT immunoreactivity intensity of IBS-total, IBS-D, IBS-M and IBS-C differed significantly from that of controls $(\mathrm{P}<0.01$, $\mathrm{P}<0.05, \mathrm{P}<0.05$ and $\mathrm{P}<0.001$, respectively).

\section{Discussion}

The rapid advances in the software for computer image analysis has rendered it possible to measure the intensity of the colour product of immunohistochemical staining for a specific intracellular substance. This intensity reflects the cellular contents of this particular substance. These measurements are useful in comparing similar tissue from different groups treated in the same conditions during immunostaining.

The present study showed that whereas the immunoreactivity intensity of serotonin in the ileum of IBS patients did not differ from that in control subjects, the immunoreactivity intensity of SERT increased regardless of the IBS subtypes. These observations were noted in the same cohort of IBS patients, where the ileal serotonin cells were lower compared with the control subjects, regardless of the IBS subtype (32). The normal cellular content of serotonin, as reflected by the immunoreactivity intensity and the reduction in the number of cells in the ileum of IBS patients implicates a decrease in the mucosal serotonin. The increase in ileal epithelial content of SERT, as indicated by increased immunoreactivity intensity, may increase the intracellular uptake of serotonin and its degradation in the gut epithelial cells and consequently decrease the availability of serotonin within the gut mucosa $(39,40)$. The present findings and the earlier observations (32) indicate that there is a decrease in the production of serotonin and a rapid degradation resulting in the low availability of serotonin at its receptors in the ileum of IBS patients.

The low availability of serotonin at it receptors in the ileum of IBS patients appear to occur in all IBS subtypes. This may indicate that this abnormality is associated with a common symptom in all IBS subtypes, which is abdominal pain/discomfort. It is possible that the abnormality in the ileum serotonin signalling system observed in the present study is responsible for the development of this common symptom in IBS patients. In agreement with this hypothesis, serotonin acts upon sensory neurons in the submucosal and myenteric ganglia as well as in spinal cord (27). The abnormality observed in this study in SERT may be genetic, as genetic abnormalities concerning SERT have been observed in IBS patients (41-50). The present observations may have an impact in the use of serotonin agonists and antagonists in the treatment of IBS patients.

\section{Acknowledgements}

The authors would like to thank Hans-Olav Fadnes for his support during the course of the study and for reading and commenting on the manuscript. This study was supported by a grant from Helse-Fonna.

\section{References}

1. Thompson WG: A world view of IBS. In: Irritable Bowel Syndrome. Camilleri M and Spiller RC (eds.) Saunders, Philadelphia and London, pp17-26, 2002.

2. Drossman DA, Li Z, Andruzzi E, et al: U.S. householder survey of functional gastrointestinal disorders. Prevalence, sociodemography, and health impact. Dig Dis Sci 38: 1569-1580, 1993.

3. El-Salhy M, Gundersen D, Hatlebakk JG and Hausken T: Irritable Bowel Syndrome: Diagnosis, Pathogenesis and Treatment Options. Nova Science Publishers, New York, 2012.

4. Ford AC and Vandvik PO: Irritable bowel syndrome. Clin Evid (Online) 2012: 0410, 2012

5. Lovell RM and Ford AC: Global prevalence of and risk factors for irritable bowel syndrome: a meta-analysis. Clin Gastroenterol Hepatol 10: 712-721, 2012.

6. Quigley EM, Locke GR, Mueller-Lissner S, et al: Prevalence and management of abdominal cramping and pain: a multinational survey. Aliment Pharmacol Ther 24: 411-419, 2006.

7. Vandvik PO, Lydersen S and Farup PG: Prevalence, comorbidity and impact of irritable bowel syndrome in Norway. Scand J Gastroenterol 41: 650-656, 2006.

8. Saito YA, Schoenfeld P and Locke GR III: The epidemiology of irritable bowel syndrome in North America: a systematic review. Am J Gastroenterol 97: 1910-1915, 2002.

9. Whitehead WE, Burnett CK, Cook EW III and Taub E: Impact of irritable bowel syndrome on quality of life. Dig Dis Sci 41: 2248-2253, 1996.

10. Agréus L, Svärdsudd K, Nyrén O and Tibblin G: Irritable bowel syndrome and dyspepsia in the general population: overlap and lack of stability over time. Gastroenterology 109: 671-680, 1995. 
11. Thompson WG and Heaton KW: Functional bowel disorders in apparently healthy people. Gastroenterology 79: 283-288, 1980.

12. Kennedy TM, Jones RH, Hungin AP, O'flanagan $\mathrm{H}$ and Kelly P: Irritable bowel syndrome, gastro-oesophageal reflux, and bronchial hyper-responsiveness in the general population. Gut 43: 770-774, 1998.

13. Talley NJ, Gabriel SE, Harmsen WS, Zinsmeister AR and Evans RW: Medical costs in community subjects with irritable bowel syndrome. Gastroenterology 109: 1736-1741, 1995.

14. Hungin AP, Whorwell PJ, Tack J and Mearin F: The prevalence, patterns and impact of irritable bowel syndrome: an international survey of 40,000 subjects. Aliment Pharmacol Ther 17: 643-650, 2003.

15. Jones R and Lydeard S: Irritable bowel syndrome in the genera population. BMJ 304: 87-90, 1992.

16. Bordie AK: Functional disorders of the colon. J Indian Med Assoc 58: 451-456, 1972

17. O'Keefe EA, Talley NJ, Zinsmeister AR and Jacobsen SJ: Bowel disorders impair functional status and quality of life in the elderly: a population-based study. J Gerontol A Biol Sci Med Sci 50: M184-M189, 1995.

18. Everhart JE and Renault PF: Irritable bowel syndrome in office-based practice in the United States. Gastroenterology 100: 998-1005, 1991.

19. Wilson S, Roberts L, Roalfe A, Bridge P and Singh S: Prevalence of irritable bowel syndrome: a community survey. Br J Gen Pract 54: 495-502, 2004.

20. Harvey RF, Salih SY and Read AE: Organic and functional disorders in 2000 gastroenterology outpatients. Lancet 1 : 632-634, 1983.

21. Spiegel BM: The burden of IBS: looking at metrics. Curr Gastroenterol Rep 11: 265-269, 2009.

22. Gralnek IM, Hays RD, Kilbourne A, Naliboff B and Mayer EA The impact of irritable bowel syndrome on health-related quality of life. Gastroenterology 119: 654-660, 2000.

23. Drossman DA, Morris CB, Schneck S, et al: International survey of patients with IBS: symptom features and their severity, health status, treatments, and risk taking to achieve clinical benefit. J Clin Gastroenterol 43: 541-550, 2009.

24. Sandler RS,Everhart JE,Donowitz M, et al: The burden of selected digestive diseases in the United States. Gastroenterology 122 1500-1511, 2002.

25. Longstreth GF, Thompson WG, Chey WD, Houghton LA, Mearin F and Spiller RC: Functional bowel disorders. Gastroenterology 130: 1480-1491, 2006.

26. Drossman DA: Rome III: the new criteria. Chin J Dig Dis 7 : $181-185,2006$

27. Mawe GM, Coates MD and Moses PL: Review article: intestinal serotonin signalling in irritable bowel syndrome. Aliment Pharmacol Ther 23: 1067-1076, 2006.

28. Erspamer V: Occurrence and distribution of 5-hydroxytryptamine (enteramine) in the living organism. Z Vitam Horm Fermentforsch 9: 74-96, 1957.

29. Chen JX, Pan H, Rothman TP, Wade PR and Gershon MD: Guinea pig 5-HT transporter: cloning, expression, distribution, and function in intestinal sensory reception. Am J Physiol 275 G433-G448, 1998.

30. Spiller R and Lam C: An update on post-infectious irritable bowel syndrome: role of genetics, immune activation, serotonin and altered microbiome. J Neurogastroenterol Motil 18: 258-268, 2012.

31. Coates MD, Mahoney CR, Linden DR, et al: Molecular defects in mucosal serotonin content and decreased serotonin reuptake transporter in ulcerative colitis and irritable bowel syndrome. Gastroenterology 126: 1657-1664, 2004.
32. El-Salhy M, Gundersen D, Hatlebakk JG and Hausken T: Abnormal endocrine cells in the ileum of patients with irritable bowel syndrome. World J Gastroenterol, 2013 (In press)

33. El-Salhy M, Gundersen D, Ostgaard H, Lomholt-Beck B, Hatlebakk JG and Hausken T: Low densities of serotonin and peptide YY cells in the colon of patients with irritable bowe syndrome. Dig Dis Sci 57: 873-878, 2012.

34. El-Salhy M, Vaali K, Dizdar V and Hausken T: Abnorma small-intestinal endocrine cells in patients with irritable bowe syndrome. Dig Dis Sci 55: 3508-3513, 2010.

35. El-Salhy M, Gundersen D, Hatlebakk JG and Hausken T: Abnormal rectal endocrine cells in patients with irritable bowel syndrome. Regul Pept, 2013 (In press).

36. Coates MD, Johnson AC, Greenwood-Van Meerveld B and Mawe GM: Effects of serotonin transporter inhibition on gastrointestinal motility and colonic sensitivity in the mouse. Neurogastroenterol Motil 18: 464-471, 2006.

37. El-Salhy M, Wendelbo I and Gundersen D: Serotonin and serotonin transporter in the rectum of patients with irritable bowel disease. Mol Med Rep 8: 451-455, 2013.

38. Spiller R, Aziz Q, Creed F, et al: Guidelines on the irritable bowel syndrome: mechanisms and practical management. Gut 56: 1770-1798, 2007.

39. Keating C, Beyak M, Foley S, et al: Afferent hypersensitivity in a mouse model of post-inflammatory gut dysfunction: role of altered serotonin metabolism. J Physiol 586: 4517-4530, 2008.

40. Coleman NS, Foley S, Dunlop SP, et al: Abnormalities of serotonin metabolism and their relation to symptoms in untreated celiac disease. Clin Gastroenterol Hepatol 4: 874-881, 2006

41. Camilleri M, Andrews CN, Bharucha AE, et al: Alterations in expression of p11 and SERT in mucosal biopsy specimens of patients with irritable bowel syndrome. Gastroenterology 132: $17-25,2007$

42. Camilleri M, Busciglio I, Carlson P, et al: Candidate genes and sensory functions in health and irritable bowel syndrome. Am J Physiol Gastrointest Liver Physiol 295: G219-G225, 2008.

43. Kumar S, Ranjan P, Mittal B and Ghoshal UC: Serotonin transporter gene (SLC6A4) polymorphism in patients with irritable bowel syndrome and healthy controls. J Gastrointestin Liver Dis 21: 31-38, 2012.

44. Park JM, Choi MG, Park JA, et al: Serotonin transporter gene polymorphism and irritable bowel syndrome. Neurogastroenterol Motil 18: 995-1000, 2006

45. Saito YA, Larson JJ, Atkinson EJ, et al: The role of 5-HTT LPR and GN $\beta 3$ 825C $>$ T polymorphisms and gene-environment interactions in irritable bowel syndrome (IBS). Dig Dis Sci 57: 2650-2657, 2012.

46. Colucci R, Blandizzi C, Bellini M, Ghisu N, Tonini M and Del Tacca M: The genetics of the serotonin transporter and irritable bowel syndrome. Trends Mol Med 14: 295-304, 2008.

47. Colucci R, Gambaccini D, Ghisu N, et al: Influence of the serotonin transporter 5HTTLPR polymorphism on symptom severity in irritable bowel syndrome. PloS One 8: e54831, 2013.

48. Faure C, Patey N, Gauthier C, Brooks EM and Mawe GM: Serotonin signaling is altered in irritable bowel syndrome with diarrhea but not in functional dyspepsia in pediatric age patients. Gastroenterology 139: 249-258, 2010.

49. Kim HJ, Camilleri M, Carlson PJ, et al: Association of distinct alpha(2) adrenoceptor and serotonin transporter polymorphisms with constipation and somatic symptoms in functional gastrointestinal disorders. Gut 53: 829-837, 2004

50. Park CS and Uhm JH: Polymorphisms of the serotonin transporter gene and G-protein $\beta 3$ subunit gene in Korean children with irritable bowel syndrome and functional dyspepsia. Gut Liver 6: 223-228, 2012. 